\title{
3D spectroscopic study of galactic rings: formation and kinematics
}

\author{
A.V. Moiseev ${ }^{*, a}$, D.V. Bizyaev ${ }^{\mathrm{b}, \mathrm{c}}$ \\ ${ }^{a}$ Special Astrophysical Observatory, Nizhnij Arkhyz, 369167 Russia \\ ${ }^{b}$ Apache Point Observatory, Sunspot, NM, USA \\ ${ }^{c}$ Sternberg Astronomical Institute, Moscow, 119992, Russia
}

\begin{abstract}
In this review we consider various ring structures that are observed in galaxies. Formation and evolution of the rings are interesting problems in studies of galactic morphology. They are related to such fundamental aspects of galactic evolution and dynamics as the nature and distribution of the dark matter in galaxies, galactic interactions and internal secular evolution of galactic substructures. A significant fraction of galactic rings forms in the disks due to gravitational torques from bar-like patterns. In contrast to this internally driven origin, the phenomenon of the polar-ring galaxies is closely connected with the processes of intergalactic interactions and merging. A rare class of collisional rings reveals the density waves triggered in the stellar and gaseous disks after a strong head-on collision with a companion. We briefly review the status of studies of gas kinematics in the rings of different origin. We stress that velocity fields of the ionized gas obtained with the Fabry-Pérot interferometers provide a very important information for these studies.
\end{abstract}

Key words: peculiar galaxies, galaxies kinematics, interacted galaxies, Fabry-Perot spectroscopy

\section{Introduction}

Direct imaging reveals high- and low-contrast annular structures in numerous galaxies. Rings are mostly observed in barred galaxies but also

\footnotetext{
${ }^{*}$ Corresponding author

Email addresses: moisav@gmail.com (A.V. Moiseev), dmbiz@apo.nmsu.edu (D.V. Bizyaev)

Preprint submitted to New Astronomy Reviews

November 2, 2018
} 
can be found in some unbarred and early-type galaxies. The linear size of these structures ranges from dozen pc for the ultracompact nuclear rings (Comerón et al., 2008) up to $100 \mathrm{kpc}$ in giant colliding systems (Ghosh \& Mapelli, 2008). The rings are often found to be the sites of active star formation and, in majority of cases, results of some collective processes in stellar-gaseous disks associated with resonance phenomena or annular density waves. Buta \& Combes (1996) presented an extensive review of ring phenomenon, first of all concerning such structures associated with bars or other non-axisymmetric disk features. In general case, rather different mechanisms are responsible for the formation of the inner and outer galactic rings. The galactic rings can be classified as follows:

1. A large class of the rings which is connected with the Lindblad's dynamical resonances in the galactic disks ('resonance rings').

2. The objects formed after a strong head-on collision with a companion ('collisional rings'). The prototype is the Cartwheel galaxy.

3. Polar rings are also results of the galaxy-to-galaxy interactions or accretion of matter with a roughly orthogonal direction of its angular moment with respect to the target galaxy. Buta \& Combes (1996) mentioned a type of 'accretion rings' which includes also objects with a coplanar orientation of the ring and main galaxy. The Hoag's object is a possible prototype.

The origin and evolution of the rings are closely connected with various aspects of galactic life. A comprehensive study of evolution of the rings should involve various observational data, among which the kinematical information is one of the most important. A panoramic (3D) spectroscopy which provides spectra for each spatial element of certain two-dimensional field of view is a powerful technique in the spatial-resolved study of kinematics in the galaxies. In this article we briefly review some results of studies of inner kinematics in the ring galaxies conducted mostly with the scanning Fabry-Pérot interferometers (FPIs). The FPI data allow to obtain the largescale velocity fields, maps of the velocity dispersion and monochromatic flux in the ionized gas simultaneously. The main goal of such observations is reconstruction, under various model assumptions, of spatial distribution of the gas velocities. Usually FPI yields the angular resolution significantly better than that obtained with the radio observations of neutral and molecular gas. This is critical when the kinematically decoupled regions are considered. Majority of the results presented in this review were obtained during 

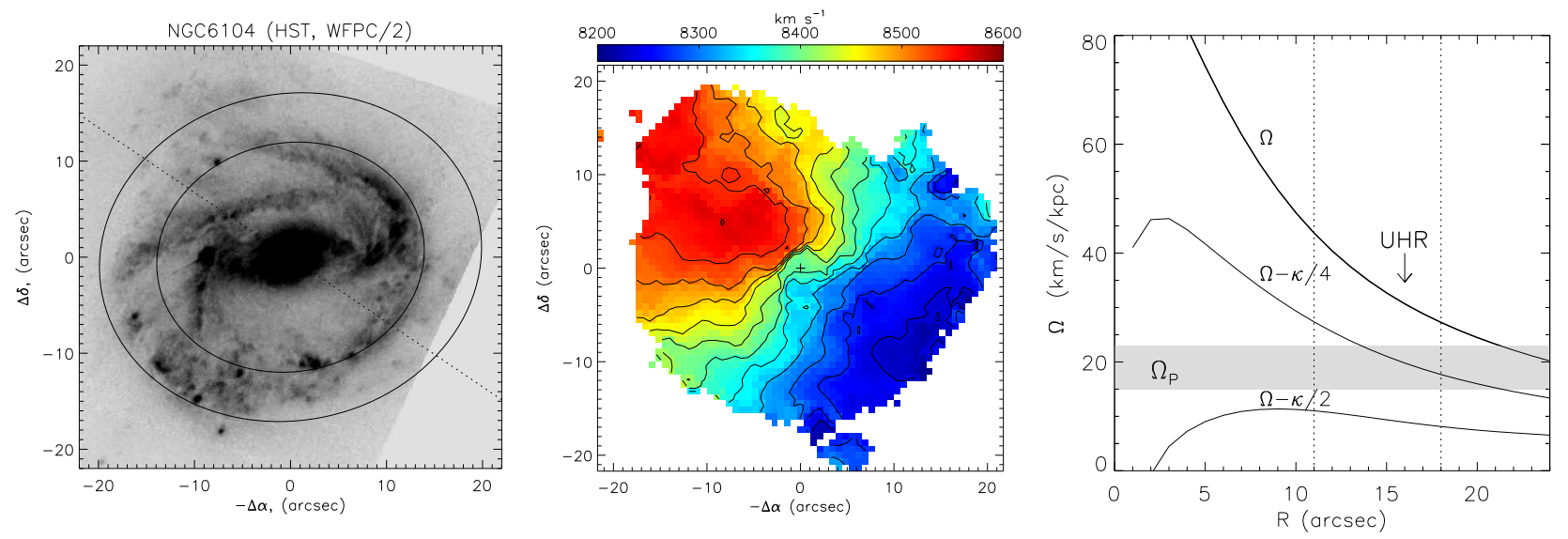

Figure 1: NGC 6104. Left: The HST/WFPC2 image deprojected to the face-on position. The dotted line corresponds to the line of nodes, the ellipses designate the ring's borders. Middle: the velocity field in the $\mathrm{H} \alpha$ emission line derived from the SCORPIO/FPI data. Right: the diagram of characteristic frequencies. The gray color designates the range of the pattern speed of the bar $\left(\Omega_{p}\right)$, the dotted lines indicate the maximum and minimum ring radii.

last decade with Russian 6-m telescope and a multi-mode spectrograph SCORPIO (Afanasiev \& Moiseev, 2005). Also we mention some results of studies of the ring kinematics obtained by several other teams that use FPI and/or integral-field devices.

\section{Resonance rings}

\subsection{A classical picture}

According to the different statistical estimations, the rings are observed in a significant fraction, up to 20-30\%, of spiral and lenticular galaxies and closely associated with the non-axisymmetric structures like bars, ovals or triaxial bulges. This fact is traditionally explained with the orbits crowding and the gas accumulation at the Lindblad's resonances (see a review by Buta \& Combes (1996)). In the terms of the theory, the empirical subdivision of the rings to the 'nuclear', 'outer' and 'inner' corresponds to the inner (ILR), outer (OLR) and ultraharmonic (UHR) dynamical resonances between the epicyclic oscillations in the stellar component and rotation of the bar. Kinematical effects observed in the resonance rings are not very prominent because the amplitude of possible non-circular gas motions (tens of $\mathrm{km} \mathrm{s}^{-1}$ ) is significantly smaller than velocities of the circular rotation 

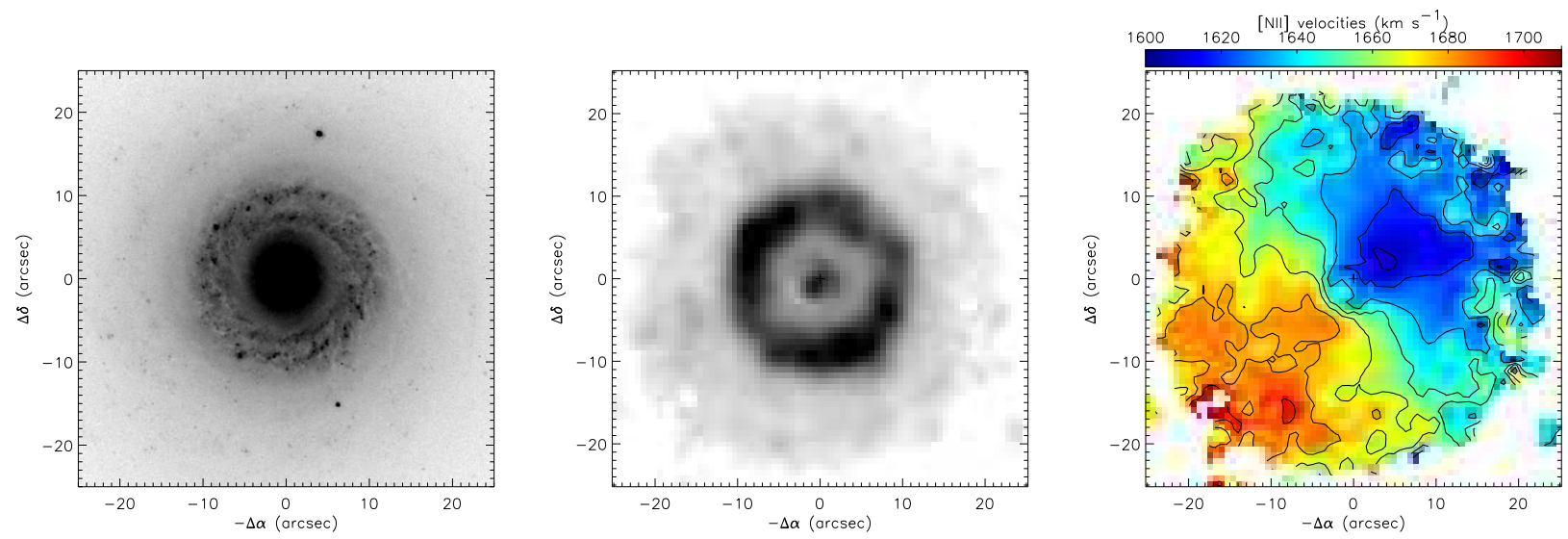

Figure 2: NGC 7742. Left: a HST/WFPC2 image. Middle: a monochromatic image in the [NII] emission line. Right: the ionized gas velocity field (Sil'chenko \& Moiseev, 2006)

$\left(150-200 \mathrm{~km} \mathrm{~s}^{-1}\right)$. It is very difficult to extract the pure rings kinematics itself. In general, it is a part of complex inward/outward motions driven by the bar potential. Interpretation of the line-of-sight velocities is often difficult because their projection depends on the assumed orientation of the galaxy disk. The high-accuracy FPI velocity field in combination with a good coverage of the filed of view (thousands of independent measurements per typical field) helps to understand the overall picture of peculiar gas motions. Long history of study of the bright star-forming ring in NGC 4736 is a good illustration. Firstly, van der Kruit (1974) from long-slit spectra suggested the idea of 'expanding H II regions ring'. Than, using the FPI data Buta (1988) concluded that the ring kinematics can be better interpreted as a result of the secular evolution in this galaxy due to its ILR. Using new TAURUS-II FPI observations Muñoz-Tuñón et al. (2004) drawn a more detailed picture which includes a radial stream driven by the bar and chaotic gas motions associated with starburst knots in the galaxy. Moiseev et al. (2004) also detected the inflow gas motions related to the bar in this galaxy.

The complex morphology of the rings+bar+spiral structure is convenient to study in moderately inclined galactic disks. Restoration of the rotation velocity fields from observations in the case of nearly face-on orientation is a serious problem which can be resolved via examination of the velocity fields under assumption of domination of the rotational component. A general analysis of both the rotation curves extracted from the FPI velocity fields and from the surface brightness distribution for several 
galaxies (each of them has more than one ring) shows that the loci of the rings agree very well with predictions from the dynamics of barred galaxies (Arsenault et al., 1988; Buta et al., 1995, 1998; Buta \& Purcell, 1998; Fathi et al., 2007). This way allows to estimate the angular speed of the bar pattern $\left(\Omega_{p}\right)$ which is one of the key characteristics for understanding the galaxy's secular evolution. The ionized gas velocity fields and observed morphological features were reproduced by numerical simulations in the works which considered IC 4214 (Salo et al., 1999) and NGC 6782 (Lin et al., 2008). The temporal evolution of their disks was studied and the pattern speed was restored as one of the main parameters in the simulations.

A model independent method of $\Omega_{p}$ estimation introduced by Tremaine \& Weinberg (1984) came into a wide usage in studies of the stellar component in early type galaxies and of the molecular disks in late type galaxies. However, some authors (Hernandez et al., 2005; Beckman et al., 2008) show that the $\mathrm{T}$-W technique (with some modification) works correctly also for the ionized gas which is an example of a media where the continuity equation gets broken. A barred Seyfert galaxy NGC 6104 is a good example for it (Smirnova et al., 2006). The pattern speed derived from the $\mathrm{H} \alpha$ velocity field agrees very well with the ring location near 1:4 ultraharmonic resonance, as it follows from the theory (Fig. 1). The $\Omega_{p}=$ const line in Fig. 1 does not intersect the $\Omega-\kappa / 2$ curve, therefore the bar has no ILR where the radial gas motions toward the center cease, so gaseous clouds can reach the center and provide a fueling for the nuclear activity.

\subsection{Rings without a bar}

The objects which are failed to be explained in the frames of a simple theory of dynamical resonances are very interesting. There are several examples of spectacular nuclear rings in unbarred galaxies. Most of these galaxies are observed face-on, so the conclusion about the bar absence is rather solid for them. Explanations for the origin of such rings include the resonance effects produced by a weakly triaxial potential employ an idea that such rings could be explained by weakly triaxial distortions, or by tidal actions from a gravitationally bounded companion, since the non-axisymmetric gravitational perturbations in these cases are similar to those from a bar (see Buta \& Combes (1996) and Sil'chenko \& Moiseev (2006) for references). Knapen et al. (2004) suggest that the latter mechanism, i.e. a recently occurred interaction with a dwarf gas-rich satellite, is able to produce the nuclear ring in the unbarred galaxy NGC 278. Their conclusion is based 
on the strongly peculiar kinematics of the neutral and ionized hydrogen detected in the observed gas velocity fields. Sil'chenko \& Moiseev (2006) proposed the same scenario for the emergence of the rings in NGC 7217 and NGC 7742 (fig. 2). Both galaxies demonstrate some signatures of recent minor-merging events in their stricture and kinematics, like the two-tiered stellar disks and mutually counterrotating subsystems. NGC 7742 has all its gas in the counterrotation with respect to the stellar component.

Using results from the integral-field spectroscopy, Mazzuca et al. (2006) also show an evidence for the tidally induced origin of the ring in NGC 7742 . This idea agrees with results of numerical simulations of the density redistribution during the galactic tidal interactions in the case if a satellite moves in the plane of the main galaxy in the direction reverse to the main rotation (Tutukov \& Fedorova, 2006).

\section{Collisional rings}

Collisional ring galaxies are results of nearly central, head-on passage of a satellite through the main, or target galaxy. Significant part of all young stars in such ring galaxies is observed in their star forming rings, and the galaxies in a total demonstrate moderately high star formation rates, like $18 \mathrm{M}_{\odot} y r^{-1}$ in the Cartwheel (Mayya et al., 2005), which is often called a prototype for this class of objects. They can be subdivided by morphological classes RE, RN or RK, named after the empty rings, rings with nuclei, or with dominant knots by Theys \& Spiegel (1976).

The collisional rings are rather rare among all ring galaxies. Thus, Madore et al. (2009) in their new Atlas and Catalog of collisional ring galaxies identified 127 rings of collisional origin from some 7000 peculiar objects. The rings classification, possible scenarios of their formation and evolution were considered by Appleton \& Struck-Marcell (1996) and modeled numerically by Lvnds \& Toomre (1976), Thevs \& Spiegel (1977), Struck-Marcell \& Higdon (1993), Mazzei et al. (1995), Athanassoula et al. (1997), Korchagin et al. (1998), Vorobyov \& Bizyaev (2003), Bizyaev et al. (2007), and by many others. All authors notice the relatively short lifetime of the collisional rings (of the order of a few hundred million years) and their transient nature. Among the three most popular explanations of the collisional ring supporting mechanisms: the material wave expanding inside out, or wave of self-inducing star formation, or propagating density wave (without a real mass transfering), the latter gets the most reliable support from observations. 
The collisional origin of the rings assumes that the ring pattern in the galaxies is not a steady structure; it should propagate through the disks. This gives us an interesting possibility to study the star formation when its different episodes are separated in time and space simultaneously since the moving front of the star formation leaves behind the progressively aging stellar population (see Bizyaev et al. (2007)). One of the key ingredient necessary for understanding the mechanism[s] of the ring formation and evolution is the picture of the kinematical motions of the gas and stars in the ring galaxies.

The expansion of the collisional rings was predicted by numerical simulations, but the ultimate confirmation of the expansion comes from the $\mathrm{HI}$ radio- and $\mathrm{H} \alpha$ optical observations focused on kinematics of the neutral and ionized gas. That makes the 3D spectroscopy an extremely important tool in studies of the collisional rings.

The kinematical studies of the gas motion in Arp 10 and the Cartwheel were performed by Fosburv \& Hawarden (1977), Charmandaris \& Appleton (1996), Higdon (1996), Amram et al. (1998). They gave out rather contradictory results on the expansion speed of the Cartwheel external ring: 13-30, 53, and $89 \mathrm{~km} / \mathrm{s}$ from the IFP $\mathrm{H} \alpha$, VLA HI and long-slit spectroscopy, respectively. Even more discrepancy emerges when the presentday ring expansion speed is compared with the secular (averaged over last few hundred Myrs) speed of the ring inferred from the color distribution (Vorobyov \& Bizvaev, 2003), and the radial distribution of spectral indices Bizyaev et al. (2007). Such a difference finds its explanation in the wave of density model of the collisional ring expansion. One more argument for this scenario is a lack of powerful shockwaves at the outer edge of the rings, as it is noticed for the case of Arp 10 (Bizyaev et al., 2007).

The FPI data, in a combination with the long-slit spectroscopy and photometry, can help to effectively investigate the history of propagating star formation in the ring galaxies, as it has been done for Arp 10. The H $\alpha$ velocity field obtained by Bizyaev et al. (2007) shows an evidence for significant radial motions in both outer and inner galactic rings. In Bizyaev et al. (2007), we fit the kinematical data with a model velocity field taking into account the circular rotation, possible radial motions and projection effects. The expansion velocity in the NW part of the outer ring in Arp 10 exceeds $100 \mathrm{~km} \mathrm{~s}^{-1}$, whereas it attains only $30 \mathrm{~km} \mathrm{~s}^{-1}$ in the SE part. This asymmetry may be a result a substantially off-centered passage of the satelliteintruder through the plane of the main galaxy. We identified the intruder 

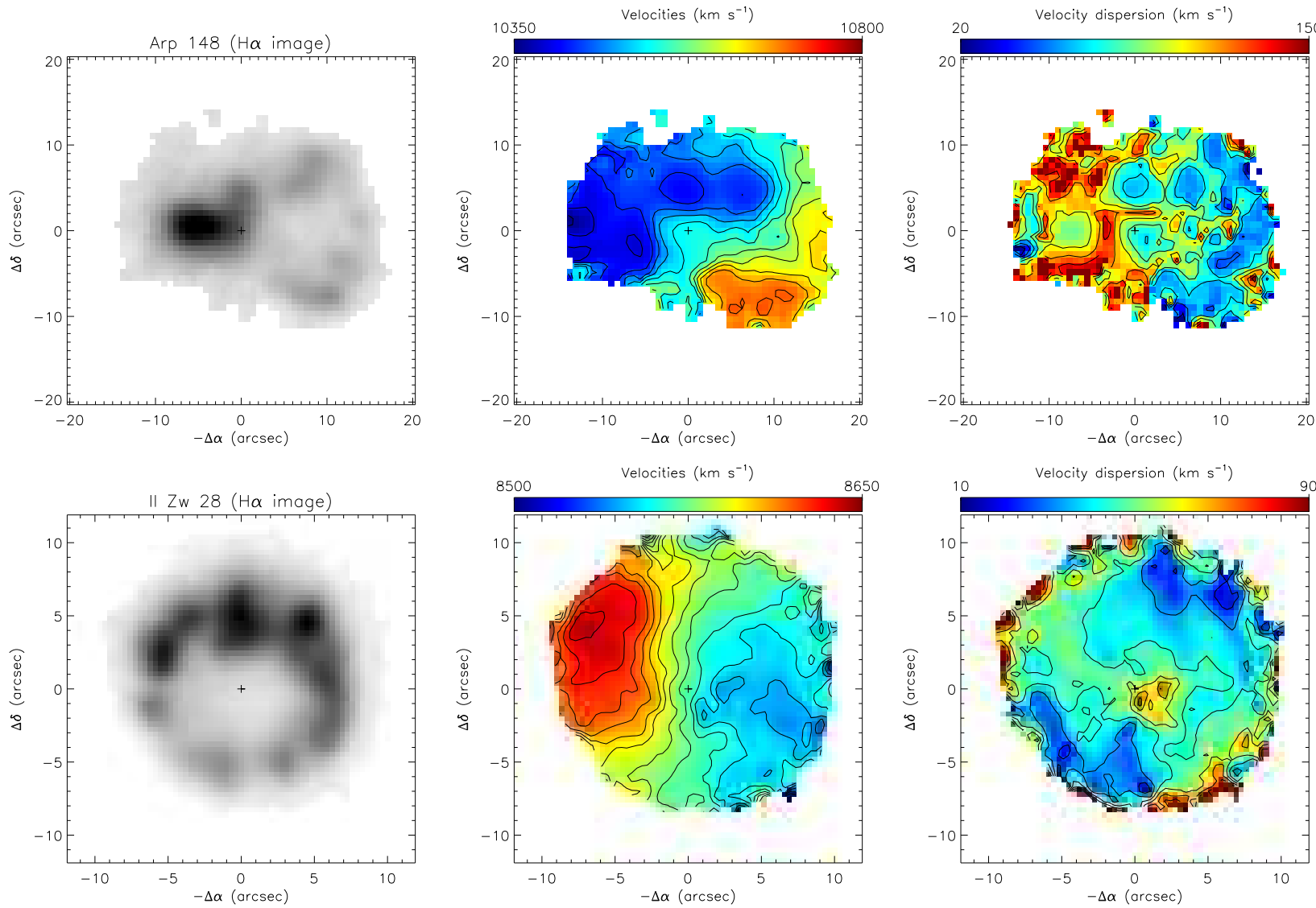

Figure 3: FPI observations of Arp 148 (top) and II Zw 28 (bottom). Left panels: the $\mathrm{H} \alpha$ images. Middle panels: the velocity fields of the ionized gas. Right panels: the maps of the ionized gas velocity dispersion.

as a former early-type spiral galaxy whose mass was about $1 / 4$ of that of the target galaxy before their collision. The size of the inner and outer rings, their expansion velocity and the radial profile of the gas circular velocity can be reproduced in our model of a rather off-center collision (the impact occurred at about $3 \mathrm{kpc}$ from the nucleus of the target galaxy) which happened approximately 85 Myr ago.

We currently run a complex program of the FPI, long-slit and photometric observations with the SAO RAS $6 \mathrm{~m}$ telescope for several more prominent northern collisional rings (Bizyaev et al., 2009) in order to constrain the evolution scenarii for the ring galaxies from the comparison of their present-day and past kinematics. We obtain patterns of the non-circular motions in the 
galaxies by subtracting the model circular motion of ionized gas from the observed $\mathrm{H} \alpha$ velocity field. Our preliminary analysis of the collisional rings shown in Fig. 3 suggests that the non-circular motions, including the radial expansion, can be identified in all considered stellar systems. At the present time, despite the valuable theoretical contributions to the understanding of the nature of the collisional ring galaxies, the observational confirmation of systematical radial motions is found for just a few objects (Cartwheel and Arp 10, see references above) with the help of radio $\mathrm{HI}$ or $\mathrm{H} \alpha$ observations. Kinematical observations of more number of the collisional rings will allow us to investigate diversity of the internal motions in this kind of galaxies.

\section{Polar rings}

\subsection{Large-scale polar rings}

The polar-ring galaxies (PRGs) are objects which are determined by the possession of the outer rings or disks of gas, dust and/or stars in them which orbit nearly orthogonally to the plane of the main galaxy. PRG are believed in most cases to be formed due to the galactic mergers and accretion of the matter from a companion galaxy or gaseous filaments from the intergalactic medium onto the host galaxy (Bournaud \& Combes (2003); Combes (2006) and references therein).

The circular rotation in two mutually perpendicular planes provides a rare chance to probe the three-dimensional mass distribution in the galaxy and to determine the spatial shape of the dark halos (Combes, 2006). To do this, it is necessary to obtain sufficiently detailed data about the inner and outer kinematics of the PRG. Whitmore et al. (1990) list 157 candidates to the polar-ring galaxies selected mostly by their peculiar appearance. However, the number of true PRG, i.e. of those which bona fide exhibit a rotation in orthogonal planes, is much smaller, about twenty galaxies. Even in the simplest case when both the central galaxy and its ring are observed edge-on, at least two long-slit spectroscopic sections are required to determine the rotation pattern in both subsystems. A number of problems arises in the case of objects where the host galaxy or ring is observed with a moderate inclination to the line-of-sight. In these cases we have to determine the two-dimensional velocity fields to study kinematic in such systems. The example of NGC 2655 is a good illustration. It was suspected to have a polar ring due to the powerful dust lane crossing the disk of the main galaxy. The comparison of the velocity fields of the gas and stars in the circumnuclear region confirmed this interpretation (Sil'chenko \& Afanasiev, 2004). 
Recently conducted 21-cm line observations allow to analyze the structure and kinematics of the polar ring (Sparke et al., 2008).

One of the first FPI data for the PRGs were briefly presented by Nicholson et al. (1987) who suggested a strong $\left(\sim 90^{\circ}\right)$ warp of the outer regions of the polar gas disk in NGC 2685. The detailed picture of a complex helical warp was presented recently with HI data (Józsa et al., 2009). Sackett \& Pogge (1995) made a conclusion about a significantly flattened dark halo in A01360801 which is based on a relationship between the elongated orbit position and FPI velocity field. Recently the St.Peterburg University's team made an attempt to observe several polar rings with panoramic spectroscopy devices and the SAO RAS 6-m telescope (Shalyapina et al., 2004; Hagen-Thorn et al., 2005; Merkulova et al., 2008).

Using the velocity fields enables to find a kinematically decoupled polar component even though it is not clearly distinguished in the morphological structure. Recent observations of Arp 212 give a good example for it (Moiseev, 2008). In the ionized gas, the two kinematically distinctive subsystems are found (Fig. 4). The first one, at $r<3.5 \mathrm{kpc}$, rotates in the plane of the stellar disk. The second system is located at $r=2-6 \mathrm{kpc}$ from the center and consists of several isolated HII regions whose orbits are tilted by high angles to the main stellar disk. Our own observing results and the data from literature on the kinematics of molecular, ionized, and neutral gas can be explained in terms of the model which implies that most of the gas in the outer parts of the galaxy concentrates in a broad ring with radius of about $20 \mathrm{kpc}$. The outer ring regions rotate in the polar plane. Once the galactocentric distance decreases, the orbits of the gaseous clouds precess and approach the galactic disk. This precession is due to the non-spherical (maybe a triaxial) shape of the gravitational potential in Arp 212.

\subsection{Nuclear polar rings and disks}

The 3D spectroscopy provides a good spatial sampling that is important for studies of the inner polar rings/disk with sizes from 100-200 pc to $1-2 \mathrm{kpc}$. Their contribution to the total luminosity of the whole circumnuclear region in their host galaxy is small. Usually the ionized gas is mostly presented at the polar orbits, however the mixed stellar-gaseous structures can also be observed. It is interesting to note that the number of confirmed inner polar structures is larger than that of the 'classical' PRGs. At the present time we count 31 objects with the inner polar/inclined disks or rings. The sample includes 17 objects listed by Corsini et al. (2003), 8 galaxies studied by Sil'chenko \& Afanasiev (2004) and a few more from 

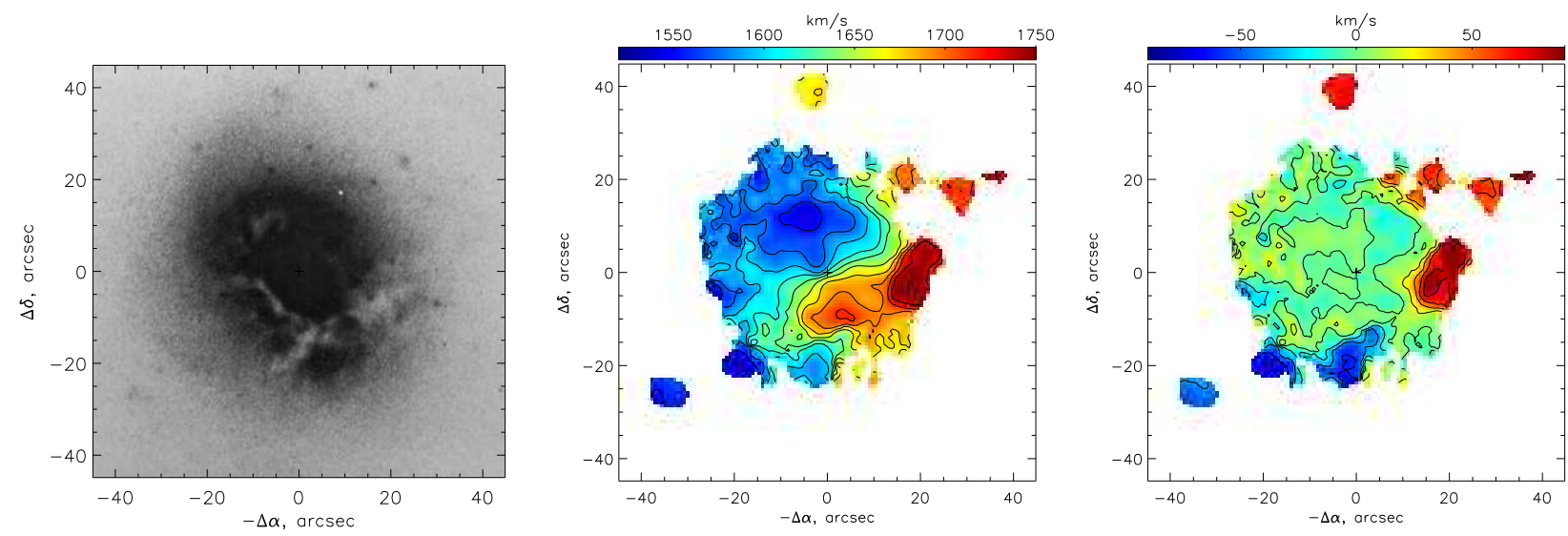

Figure 4: Arp 212. Left: a red-plate photo from the Arp's atlas. Middle: $\mathrm{H} \alpha$ velocity field obtained with the SCORPIO/FPI. Right: the residual velocity field (after subtraction of the model circular field found out from the rotation of the inner disk) reveals the kinematically decoupled regions which are seen as knots in the warped polar ring

other papers, see some references in Sil'chenko et al. (2009). The major fraction of the nuclear polar structures has been confirmed with the 3D spectroscopy (integral-field or FPI) with the SAO RAS 6-m telescope. Detailed observations reveal that in some cases the inner polar orbits extend down to the equatorial plane at the large radii, as it can be seen from the VLT/VIMOS data for NGC 2685 (Coccato et al., 2007). The smooth transition from the polar to the 'normal' orientation of rotation is exhibited by the ionized gas in late-type galaxy NGC 7468 observed with the SCORPIO/FPI by Shalyapina et al. (2004).

The origin of the inner polar structures is not well understood yet. The most popular hypothesis is the external gas accretion as for a large-scale PRG. However, some dynamical mechanisms of their formation are also proposed, like a transportation of the gas onto highly-inclined anomalous orbits by a tumbling triaxial potential, see Sil'chenko \& Afanasiev (2004) for detailed discussion. More precise observations and numerical simulations are required to explain successfully these galactic structures. We hope that increasing amounts of observational information about the inner polar disks will stimulate new theoretical efforts in this direction.

This review is based on observations made with the 6-m telescope at the Special Astrophysical Observatory of the Russian Academy of Sciences, which is operated under the financial support of the Ministry of Science of 
the Russian Federation (registration number 01-43). A.M. thanks the 7th SCSLSA Organized Committee for their kind invitation and hosting. This work was supported by the Russian Foundation for Basic Research (project no. 09-02-00870)

\section{References}

Afanasiev, V. L., Moiseev, A. V., 2005. Astronomy Letters 31, 194.

Amram, P., Mendes de Oliveira, C., Boulesteix, J., Balkowski, C., 1998. A\&A 330, 881. Appleton, P. N., Struck-Marcell, C., 1996. Fundamentals of Cosmic Physics 16, 111. Arsenault, R., Boulesteix, J., Georgelin, Y., Roy, J.-R., 1988. A\&A 200, 29.

Athanassoula, E., Puerari, I., Bosma, A., 1997. MNRAS 286, 284.

Beckman, J., Fathi, K., Piñol, N., Hernandez, O., Carignan, C., Pérez, I., 2008. To be published in MSAIt. arXiv:0810.3996 [astro-ph].

Bizyaev, D., Moiseev, A., Vorobyov, E., 2009. In: Bulletin of the American Astronomical Society. Vol. 41. p. 328.

Bizyaev, D. V., Moiseev, A. V., Vorobyov, E. I., 2007. ApJ 662, 304.

Bournaud, F., Combes, F., 2003. A\&A 401, 817.

Buta, R., 1988. ApJs 66, 233.

Buta, R., Alpert, A. J., Cobb, M. L., Crocker, D. A., Purcell, G. B., 1998. AJ 116, 1142.

Buta, R., Combes, F., 1996. Galactic Rings. Fundamentals of Cosmic Physics 17, 95.

Buta, R., Purcell, G. B., 1998. AJ 115, 484.

Buta, R., Purcell, G. B., Crocker, D. A., 1995. AJ 110, 1588.

Charmandaris, V., Appleton, P. N., 1996. ApJ 460, 686.

Coccato, L., Corsini, E. M., Pizzella, A., Bertola, F., 2007. A\&A 465, 777.

Combes, F., 2006. In: Mamon, G. A., Combes, F., Deffayet, C., Fort, B. (Eds.), EAS Publications Series. Vol. 20. p. 97.

Comerón, S., Knapen, J. H., Beckman, J. E., Shlosman, I., Feb. 2008. A\&A 478, 403.

Corsini, E. M., Pizzella, A., Coccato, L., Bertola, F., 2003. A\&A 408, 873.

Fathi, K., Beckman, J. E., Zurita, A., Relaño, M., Knapen, J. H., Daigle, O., Hernandez, O., Carignan, C., 2007. A\&A 466, 905.

Fosbury, R. A. E., Hawarden, T. G., 1977. MNRAS 178, 473.

Ghosh, K. K., Mapelli, M., May 2008. MNRAS 386, L38.

Hagen-Thorn, V. A., Shalyapina, L. V., Karataeva, G. M., Yakovleva, V. A., Moiseev, A. V., Burenkov, A. N., 2005. Astronomy Reports 49, 958.

Hernandez, O., Wozniak, H., Carignan, C., Amram, P., Chemin, L., Daigle, O., 2005. ApJ 632, 253.

Higdon, J. L., 1996. ApJ 467, 241.

Józsa, G. I. G., Oosterloo, T. A., Morganti, R., Klein, U., Erben, T., 2009. A\&A 494, 489.

Knapen, J. H., Whyte, L. F., de Blok, W. J. G., van der Hulst, J. M., 2004. A\&A 423, 481.

Korchagin, V., Mayya, Y. D., Vorobyov, E. I., Kembhavi, A. K., 1998. ApJ 495, 757.

Lin, L.-H., Yuan, C., Buta, R., 2008. ApJ 684, 1048.

Lynds, R., Toomre, A., 1976. ApJ 209, 382.

Madore, B. F., Nelson, E., Petrillo, K., 2009. ApJs 181, 572. 
Mayya, Y. D., Bizyaev, D., Romano, R., Garcia-Barreto, J. A., Vorobyov, E. I., 2005. ApJl 620, L35.

Mazzei, P., Curir, A., Bonoli, C., 1995. AJ 110, 559.

Mazzuca, L. M., Sarzi, M., Knapen, J. H., Veilleux, S., Swaters, R., 2006. ApJl 649, L79.

Merkulova, O. A., Shalyapina, L. V., Yakovleva, V. A., Karataeva, G. M., 2008. Astronomy Letters 34,542 .

Moiseev, A. V., 2008. Astrophysical Bulletin 63, 201. arXiv: 0808.1696 [astro-ph]

Moiseev, A. V., Valdés, J. R., Chavushyan, V. H., 2004. A\&A 421, 433.

Muñoz-Tuñón, C., Caon, N., Aguerri, J. A. L., 2004. AJ 127, 58.

Nicholson, R. A., Taylor, K., Sparks, W. B., Bland, J., 1987. In: de Zeeuw, P. T. (Ed.), Structure \& Dynamics of Elliptical Galaxies. Vol. 127 of IAU Symposium. p. 415.

Sackett, P. D., Pogge, R. W., 1995. In: Holt, S. S., Bennett, C. L. (Eds.), Dark Matter. Vol. 336 of American Institute of Physics Conference Series. p. 141.

Salo, H., Rautiainen, P., Buta, R., Purcell, G. B., Cobb, M. L., Crocker, D. A., Laurikainen, E., 1999. AJ 117, 792.

Shalyapina, L. V., Moiseev, A. V., Yakovleva, V. A., Hagen-Thorn, V. A., Barsunova, O. Y., 2004. Astronomy Letters 30, 583.

Sil'chenko, O. K., Afanasiev, V. L., 2004. AJ 127, 2641.

Sil'chenko, O. K., Moiseev, A. V., 2006. AJ 131, 1336.

Sil'chenko, O. K., Moiseev, A. V., Afanasiev, V. L., 2009. ApJ 694, 1550.

Smirnova, A. A., Moiseev, A. V., Afanasiev, V. L., 2006. Astronomy Letters 32, 520.

Sparke, L. S., van Moorsel, G., Erwin, P., Wehner, E. M. H., 2008. AJ 135, 99.

Struck-Marcell, C., Higdon, J. L., 1993. ApJ 411, 108.

Theys, J. C., Spiegel, E. A., 1976. ApJ 208, 650.

Theys, J. C., Spiegel, E. A., 1977. ApJ 212, 616.

Tremaine, S., Weinberg, M. D., 1984. ApJl 282, L5.

Tutukov, A. V., Fedorova, A. V., 2006. Astronomy Reports 50, 785.

van der Kruit, P. C., 1974. ApJ 188, 3.

Vorobyov, E. I., Bizyaev, D., 2003. A\&A 400, 81.

Whitmore, B. C., Lucas, R. A., McElroy, D. B., Steiman-Cameron, T. Y., Sackett, P. D., Olling, R. P., 1990. AJ 100, 1489. 